\title{
Corrigendum: Experimental realization of an optical second with strontium lattice clocks
}

R. Le Targat, L. Lorini, Y. Le Coq, M. Zawada, J. Guéna, M. Abgrall, M. Gurov, P. Rosenbusch, D.G. Rovera, B. Nagórny, R. Gartman, P.G. Westergaard, M.E. Tobar, M. Lours, G. Santarelli, A. Clairon, S. Bize, P. Laurent, P. Lemonde \& J. Lodewyck

Nature Communications 4:2109 doi:10.1038/ncomms3109 (2013); Published 9 Jul 2013; Updated 31 Oct 2013

In the legend to Fig. $4 \mathrm{c}$ in this Article, references 47 and 48 were incorrectly included in a list of sources for ${ }^{87} \mathrm{Sr}$ clock frequency measurements. The correct sources are Hong et al. ${ }^{49}$ and Yamaguchi et al. ${ }^{50}$, as follows.

49. Hong, F.-L. et al. Measuring the frequency of a Sr optical lattice clock using a $120 \mathrm{~km}$ coherent optical transfer. Opt. Lett. 34, 692-694 (2009).

50. Yamaguchi, A. et al. Stability transfer between two clock lasers operating at different wavelengths for absolute frequency measurement of clock transition in ${ }^{87} \mathrm{Sr}$. Appl. Phys. Express 5, 022701 (2012). 